\title{
Bei Arthrosetherapie auf Begleiterkrankungen achten!
}

Patienten mit Kniegelenkarthrose sind in der Regel älter und haben häufig kardiovaskuläre Begleiterkrankungen. Diesem Umstand sollten Orthopäden bei der medikamentösen Therapie der Arthrose mehr Beachtung schenken.

Ein „Wandel im klassischen orthopädischen Vorgehen" vollzieht sich nach Dr. Johannes Flechtenmacher, Präsident des deutschen Berufsverbands für Orthopädie und Unfallchirurgie, in der Behandlung der Gonarthrose. Man müsse sich heute bewusst sein, so der Experte, dass man „nicht die Arthrose per se behandelt, sondern meist ältere Patienten mit Arthrose und Komorbiditäten".

$\mathrm{Zu}$ den in der orthopädischen Praxis am häufigsten verordneten Medikamenten gehört laut Flechtenmacher Ibuprofen. Von diesem müsse man jedoch wissen, dass es die Wirkung von Aetylsalicylsäure (ASS) aufhebt. „Patienten, die Aspirin zur Antikoagulation einnehmen, sollten kein Ibuprofen erhalten", warnte der Experte. Gegebenenfalls solle man auf ein anderes Medikament umsetzen.

\section{Kein Diclofenac bei kardiovasku- lärem Risiko}

In der aktuellen Leitlinie der OARSI (Osteoarthritis Research Society International) wird insbesondere bei Patienten mit kardiovaskulären Begleiterkrankungen oder Gebrechlichkeit von der Einnahme oraler NSAR gegen Arthroseschmerzen abgeraten. Diese

Quelle: Dr. Elke Oberhofer, SpringerMedizin.de. Basierend auf: Flechtenmacher J. Ortho Trauma Update, 6./7. März 2020, Berlin ein Rote-Hand-Brief der EMA (Europäische Arzneimittelagentur), basierend auf den Daten von 10 Mio. Versicherten, vor dem Einsatz von oralem Diclofenac bei Patienten mit Herzinsuffizienz der NYHA-Klassen II bis IV, ischämischer Herzerkrankung, peripherer Arterienerkrankung oder zerebrovaskulärer Erkrankung. Ein Jahr später war die Häufigkeit der Diclofenac-Verordnungen in Deutschland gegenüber 2011 zwar deutlich zurückgegangen. „Trotzdem", so Flechtenmacher, ,, hatten weiterhin $12 \%$ der Patienten mit erstmaliger Diclofenac-Verschreibung eine der neuen kardiovaskulären Kontraindikationen."

》) Patienten, die Aspirin zur Antikoagulation einnehmen, sollten kein Ibuprofen erhalten

Laut Flechtenmacher am zweithäufigsten verordnet wird in der orthopädischen Praxis Metamizol. Dieses sei zur Behandlung von Arthroseschmerzen aber gar nicht zugelassen. Wer es doch anwende, sollte auf eine gute Dokumentation achten und die Patienten vor allem über die mögliche Folge einer Agranulozytose aufklären.

\section{Lokalanästhetika wirken chondrotoxisch}

Ernst zu nehmen ist dem Experten zufolge auch die zunehmende Evidenz für die chondrotoxische Wirkung von Lokalanästhetika. Daher sei vor allem die kontinuierliche Injektion über Schmerzpumpen ins Gelenk zu vermeiden.

Bei Patienten mit kardiovaskulären Begleiterkrankungen und Kniearthrose (nicht Hüft- oder Polyarthrose!) empfiehlt die Leitlinie alternativ die intra-artikuläre Injektion von Hyaluronsäure oder Kortikosteroiden, gegebenfalls auch kombiniert. Gerade Letztere sollten aber möglichst nicht zusammen mit Lokalanästhetika verabreicht werden: „Wenn Sie das kombinieren, haben Sie gleich zwei toxische Substanzen", warnte Flechtenmacher.

Wie der Orthopäde betonte, ist „das Herzstück aller aktuellen Leitlinien zur Arthrosebehandlung die Patientenschulung mit dem Fokus auf strukturierte landbasierte Übungsprogramme“. In dieser Hinsicht sei die ambulante Versorgung in Deutschland "noch deutlich verbesserungsbedürftig".

Hinweis des Verlags. Der Verlag bleibt in Hinblick auf geografische Zuordnungen und Gebietsbezeichnungen in veröffentlichten Karten und Institutsadressen neutral.

rheuma plus $2020 \cdot 19: 138$ https://doi.org/10.1007/s12688020-00343-5

(๑) Springer-Verlag GmbH Austria, ein Teil von Springer Nature 2020 